\title{
A Mechanistic Understanding of Non-Classical Crystal Growth in Hydrothermally Synthesized Sodium Yttrium Fluoride Nanowires
}

Alexander B. Bard, ${ }^{\dagger}$ Xuezhe Zhou, ${ }^{\ddagger}$ Xiaojing Xia, ${ }^{\circledR}$ Guomin Zhu, ${ }^{\S}, \ddagger$ Matthew B. Lim, ${ }^{\ddagger}$ Seung Min Kim," Matthew C. Johnson, ${ }^{\perp}$ Justin M. Kollman, ${ }^{\perp}$ Matthew A. Marcus, ${ }^{\#}$ Steven R. Spurgeon, ${ }^{@}$ Daniel E. Perea, ${ }^{\triangle}$ Arun Devaraj, ${ }^{\S}$ Jaehun Chun, ${ }^{\S}$ James J. De Yoreo, ${ }^{\S, \downarrow, \dagger}$ and Peter J. Pauzauskie*, ${ }^{, \pm, \S}$

† Department of Chemistry, University of Washington, Seattle, WA 98195, USA

‡ Department of Materials Science and Engineering, University of Washington, Seattle, WA 98195, USA

II Department of Molecular Engineering and Science, University of Washington, Seattle, WA 98195, USA

$\S$ Physical and Computational Sciences Directorate, Pacific Northwest National Laboratory, Richland, WA 99352, USA

II Institute of Advanced Composite Materials, Korea Institute of Science and Technology, Jeonbuk 565-905, Republic of Korea

$\perp$ Department of Biochemistry, University of Washington, Seattle, WA 98195, USA

\# Advanced Light Source, Lawrence Berkeley National Laboratory, Berkeley, CA 94720, USA

@ Energy and Environment Directorate, Pacific Northwest National Laboratory, Richland, WA 99352, USA

$\Delta$ Environmental Molecular Sciences Laboratory, Earth and Biological Sciences Directorate, Pacific Northwest National Laboratory, Richland, WA 99352, USA

Figure S1: STEM-EDS analysis of mixed- $\alpha$ nucleated $\beta$ particles (percentages in atomic percent with respect to total rare earth concentration)

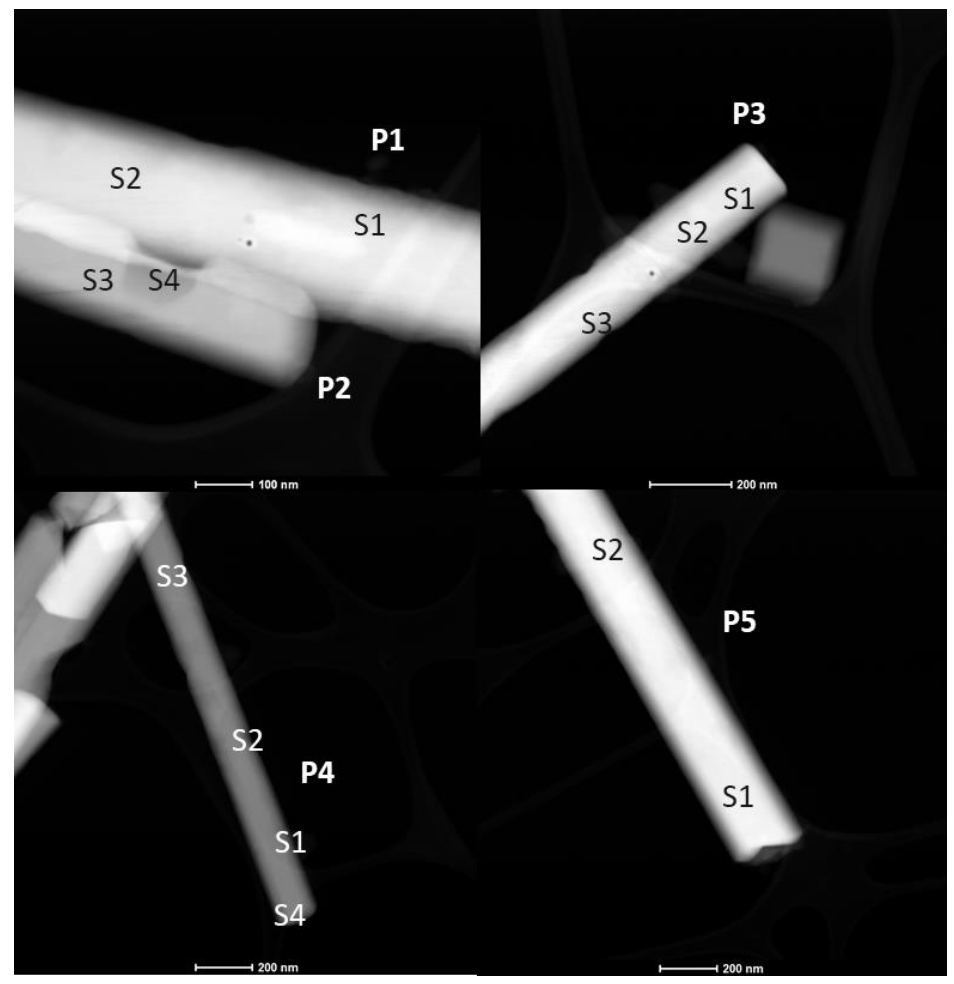

\begin{tabular}{|c|c|c|c|c|}
\hline Particle & Spot & Y\% & Yb\% & Er\% \\
\hline \multirow{2}{*}{ P1 } & S1 & 93.3 & 6.16 & 0.5 \\
\cline { 2 - 5 } & S2 & 94.4 & 4.88 & 0.8 \\
\hline \multirow{2}{*}{ P2 } & S1 & 98.4 & 1.32 & 0.3 \\
\cline { 2 - 5 } & S2 & 97.1 & 2.73 & 0.2 \\
\hline \multirow{4}{*}{ P3 } & S1 & 98.8 & 0.26 & 1 \\
\cline { 2 - 5 } & S2 & 98.7 & 0.47 & 0.9 \\
\cline { 2 - 5 } & S3 & 90.6 & 7.8 & 1.6 \\
\hline \multirow{4}{*}{ P4 } & S1 & 94.7 & 5.26 & 0 \\
\cline { 2 - 5 } & S2 & 91.6 & 5.32 & 3.1 \\
\cline { 2 - 5 } & S3 & 94.7 & 4.72 & 0.6 \\
\cline { 2 - 5 } & S4 & 90.3 & 8.15 & 1.6 \\
\hline \multirow{2}{*}{ P5 } & S1 & 89.5 & 8.8 & 1.7 \\
\cline { 2 - 5 } & S2 & 90.1 & 8.16 & 1.7 \\
\hline
\end{tabular}


Figure S2: Simulations of SAED patterns of various orientations of potential particles in the synthesis vessel with similar $d$ spacings compared to experimental data

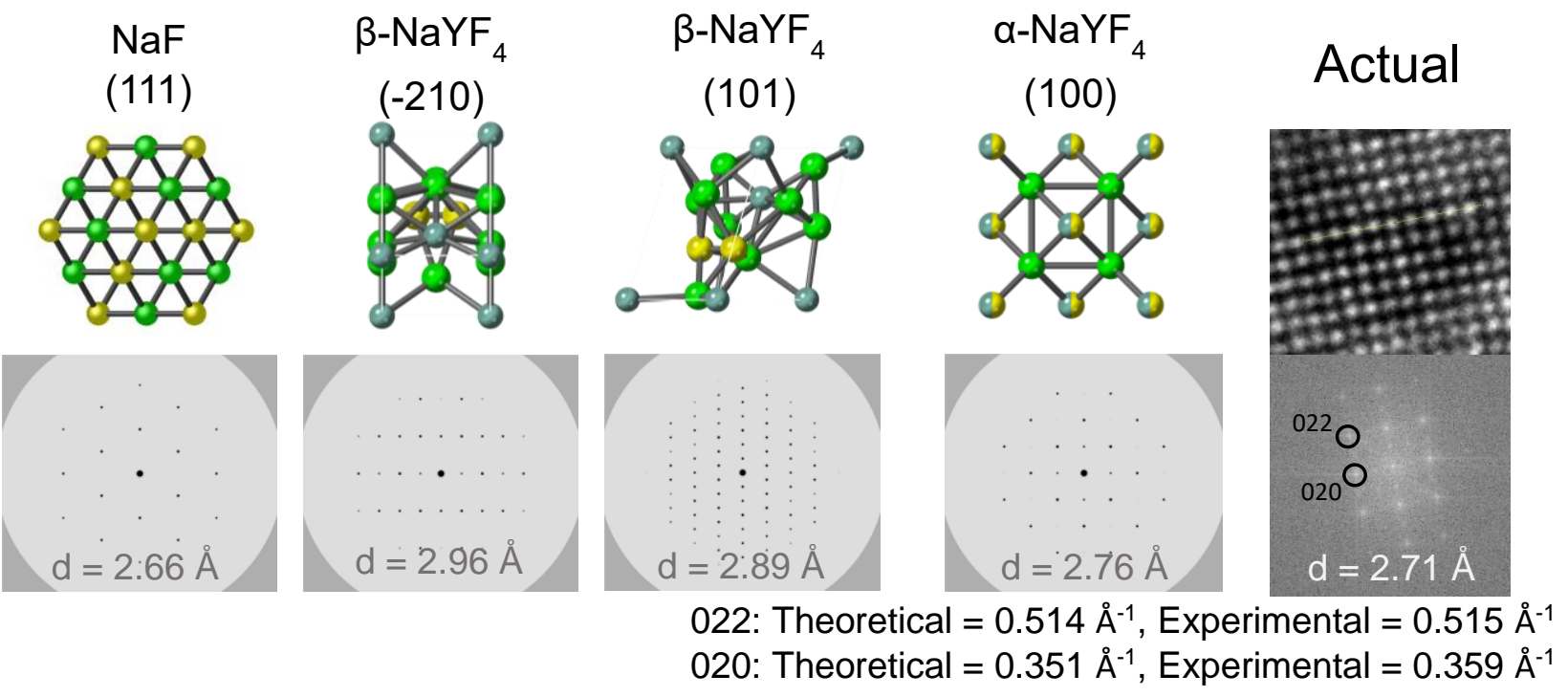

Figure S3: Mass Spectrum via Atom Probe Tomography of the $\beta-\mathrm{NaYF}_{4}$ end cap

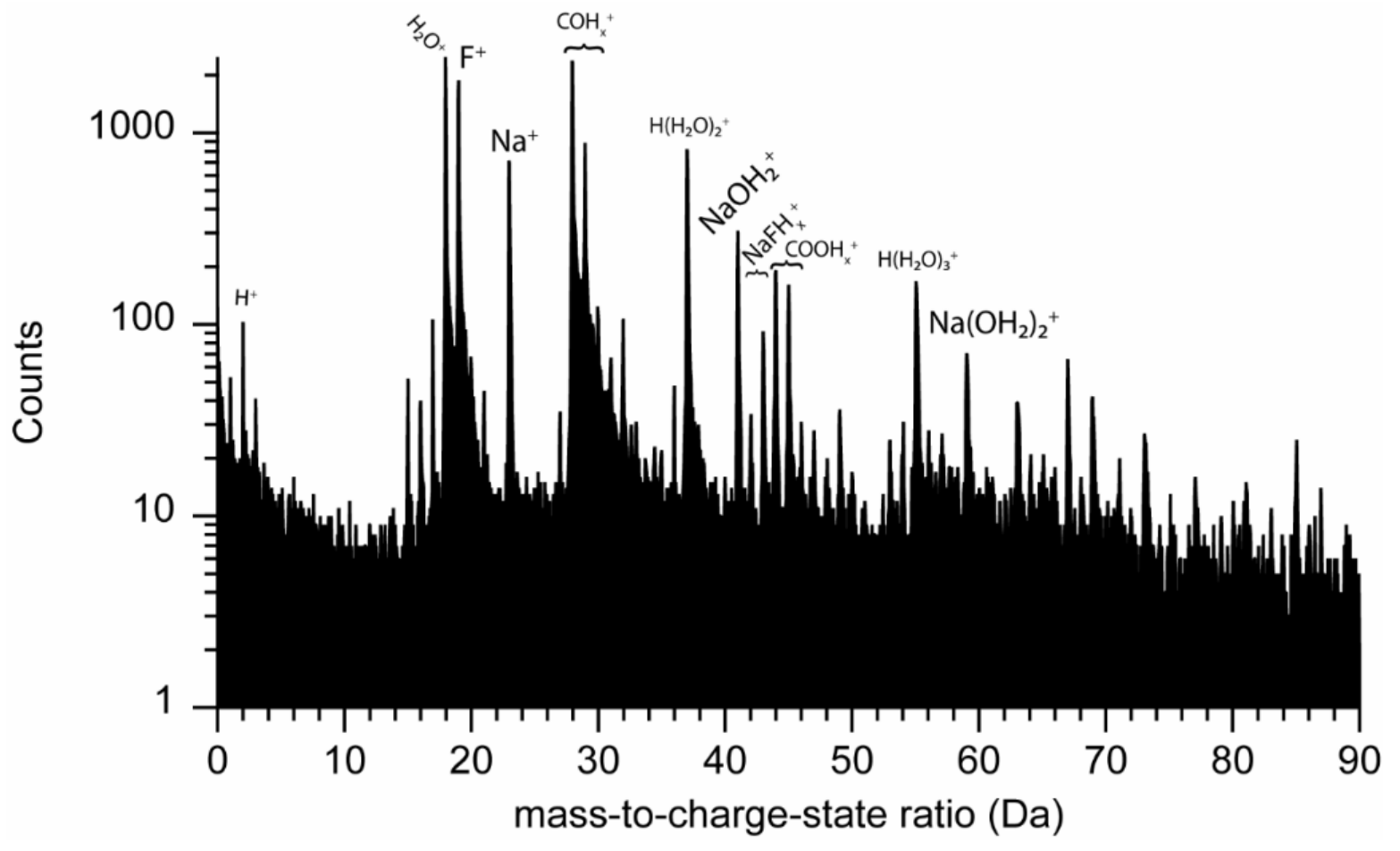


Table S4: DLS characterization of $\mathrm{NaYF}_{4}$ particles

\begin{tabular}{|l|l|l|}
\hline & Average hydrodynamic radius $(\mathrm{nm})$ & Average Zeta Potential $(\mathrm{mV})$ \\
\hline$\alpha$ (cubic) & 326.9 & -71.0 \\
\hline$\beta$ (hexagonal) & 750.8 & +18.7 \\
\hline
\end{tabular}

Figure S4: Sample DLS data

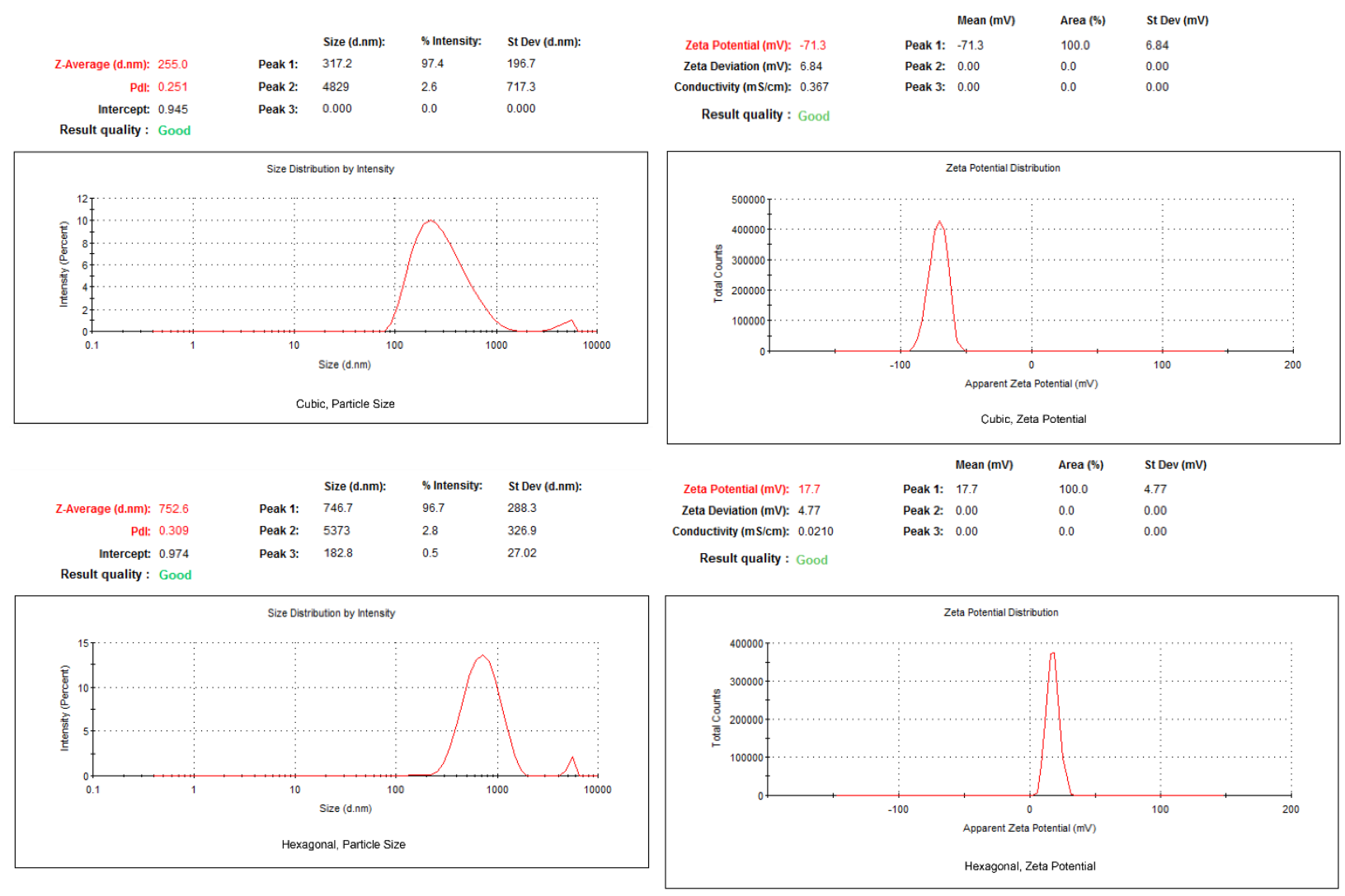

Representative DLS data. Many scans were averaged in Table S4, so numbers vary slightly. 
Figure S5: FTIR of $\mathrm{NaYF}_{4}$ particles before and after calcination

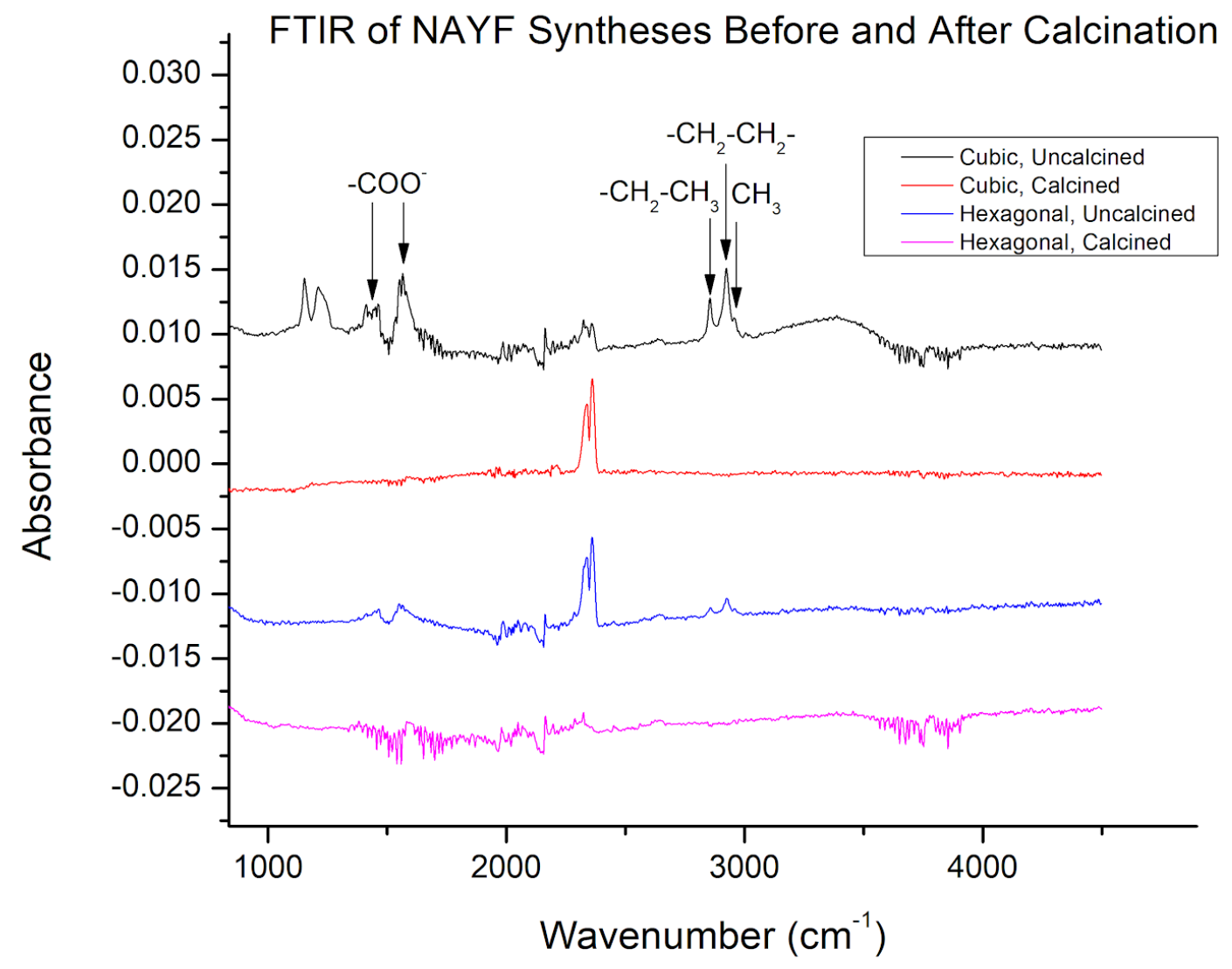

Experimental: Fourier-Transform Infrared (FTIR) spectra were taken on a Bruker VERTEX 70v FTIR Spectrometer in attenuated total reflectance (ATR) mode. Powdered $\mathrm{NaYF}_{4}$ samples were placed on the diamond ATR crystal and 16 scans were averaged. The spectral resolution of the instrument is $2 \mathrm{~cm}^{-1}$. 
Figure S6: TEM of faceted $\alpha-\mathrm{NaYF}_{4}$ clusters
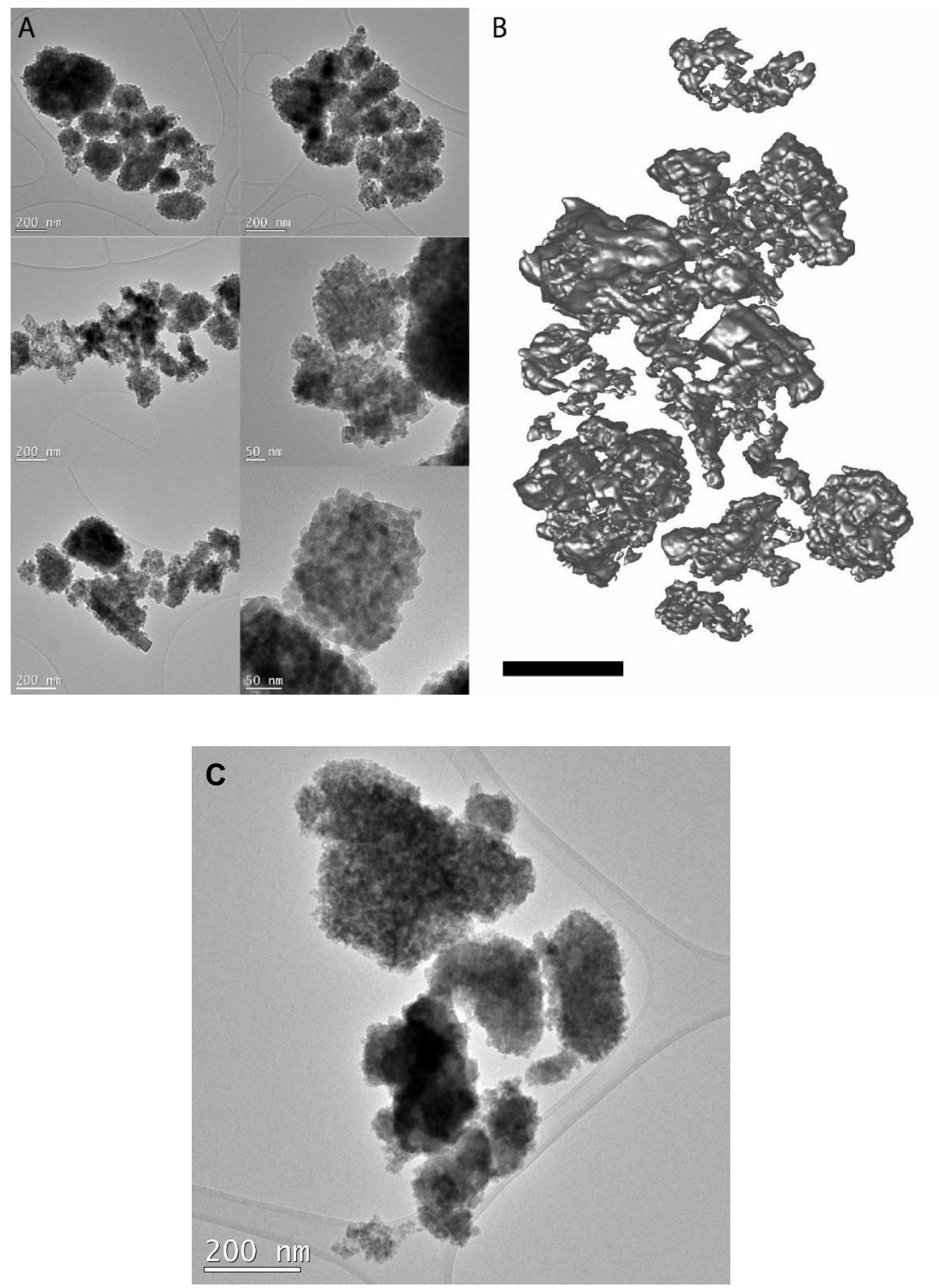

(A) Multiple clusters of $\alpha-\mathrm{NaYF}_{4}$ showing clear mesoscale faceting.at the three hour time point (B) Tomographic reconstruction of several $\alpha-N_{a Y F}$ clusters. Scale $=200 \mathrm{~nm}$. (C) Cluster of $\alpha-$ $\mathrm{NaYF}_{4}$ showing mesoscale faceting at 100 minute time point 
Figure S7: TEM of $\alpha-\mathrm{NaYF}_{4}$ Mesocrystals
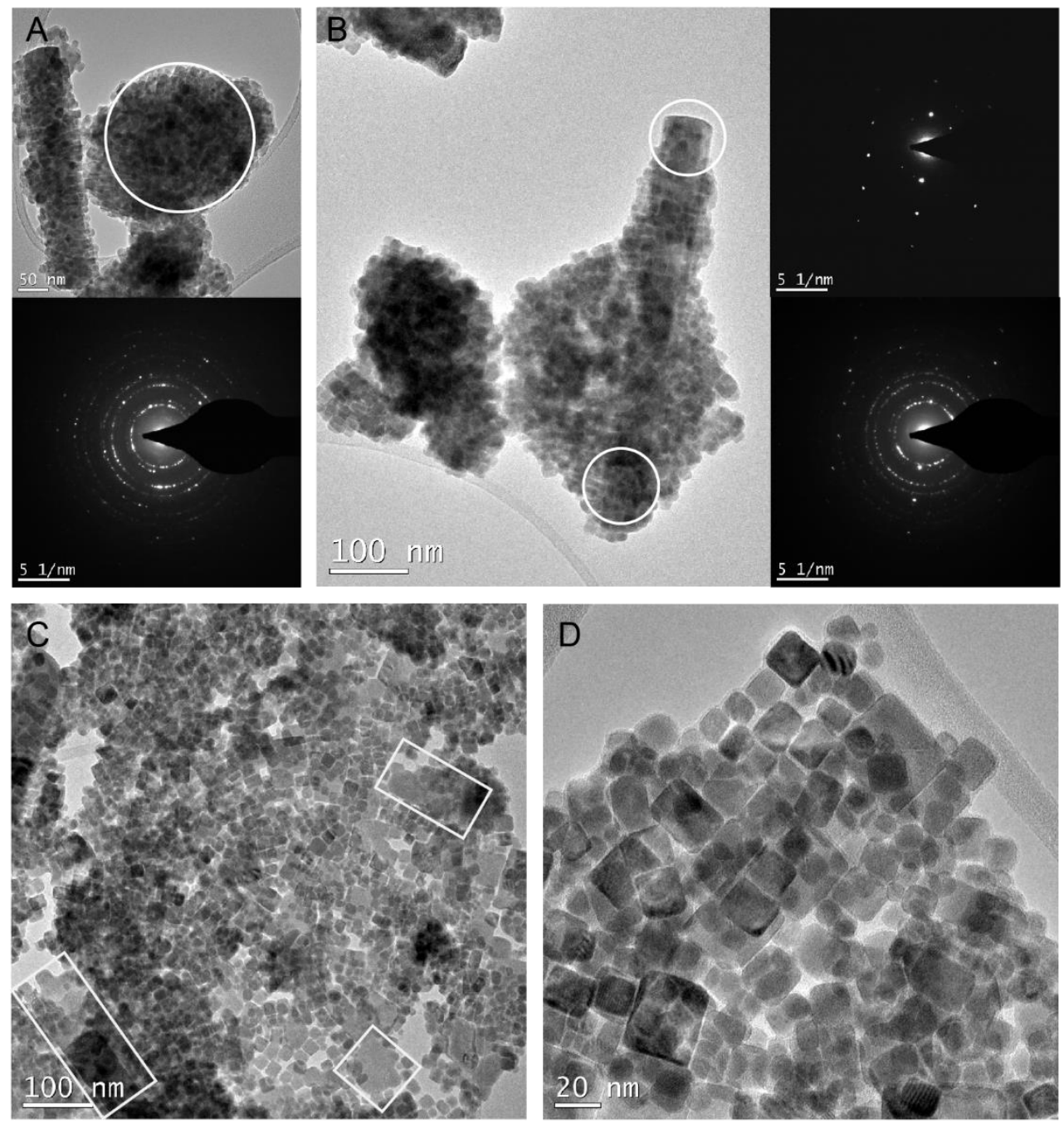

(A) TEM and SAED of an $\alpha-\mathrm{NaYF}_{4}$ cluster from the three hour synthesis showing orientation along multiple axes. Also note the single $\beta-\mathrm{NaYF}_{4}$ nanowire growing inside a cluster of small $\alpha$ $\mathrm{NaYF}_{4}$. Circled region is approximate area of SAED. (B) $\beta-\mathrm{NaYF}_{4}$ nanowire growing from a clearly faceted $\alpha-\mathrm{NaYF}_{4}$ cluster with SAED. Note crystallographic orientation of both species in bottom SAED. (C) Two dimensional layer of $\alpha-\mathrm{NaYF}_{4}$ from the same synthesis showing clear mesoscopic order. Highlighted particles appear to have undergone oriented attachment. (D) Zoom on mesoscopically ordered $\alpha-\mathrm{NaYF}_{4}$. 
Figure S8: Liquid cell TEM of cubic $\mathrm{NaYF}_{4}$ particles suspended in water aggregating due to the electron beam.
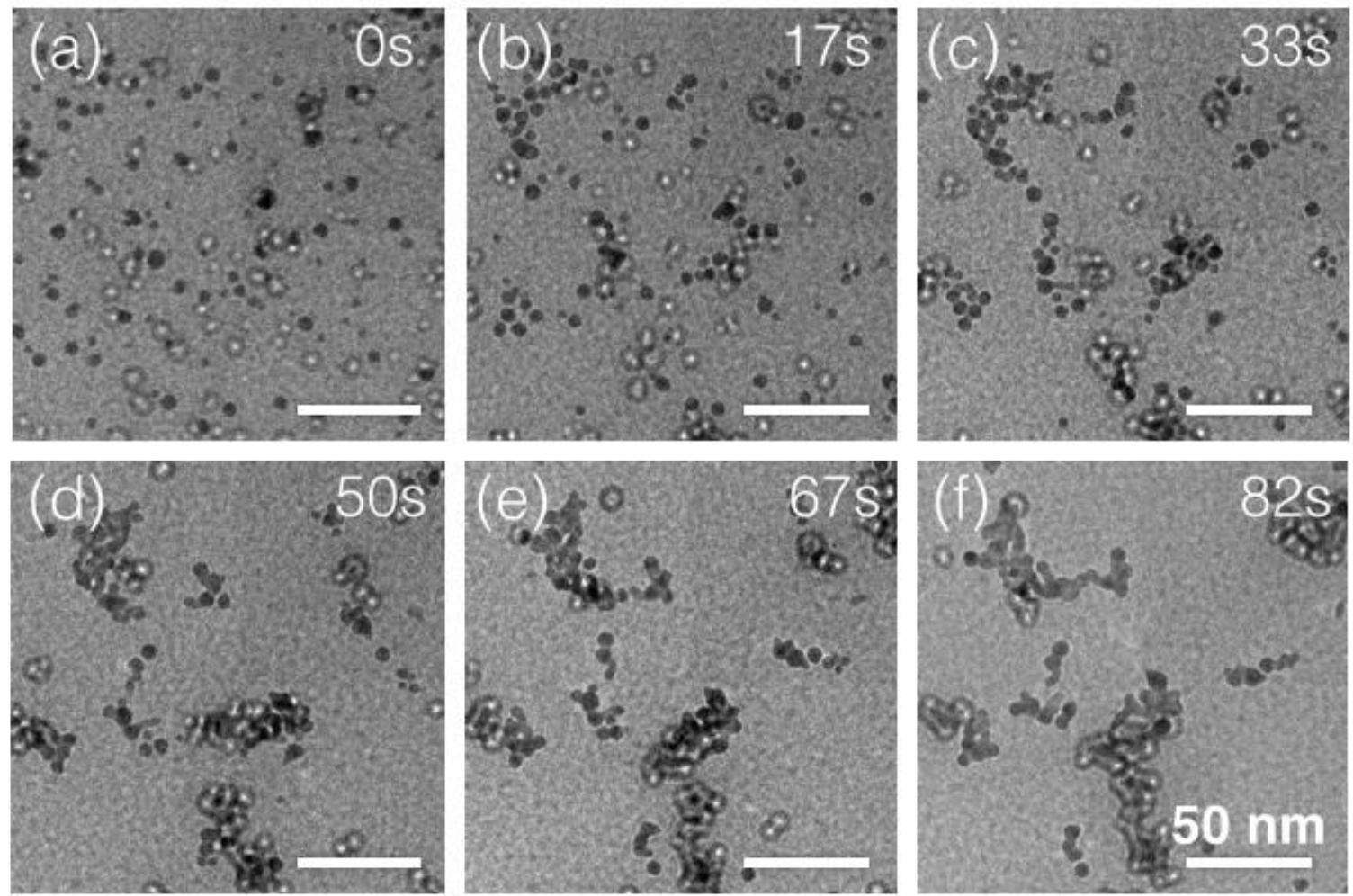

\section{EXAFS Structural Analysis: Further details}

\section{Data reduction to $k^{3} \chi(k)$}

The transmission edge jumps were about 0.025 and 0.05 optical density units. Fluorescence count rates were $70 \mathrm{kHz}$ and $100 \mathrm{kHz}$ near the edge. The tall white-line peak at the edge is sensitive to overabsorption, so it was possible to use the transmission data (assumed free of this effect) as a template with which to apply a simple correction to the fluorescence data. The resulting EXAFS data was good to about $14 \AA^{-1}$.

\section{Model for $\mathbf{P} \overline{6}$ crystal structure}

We assume that the $\mathrm{Yb}$ is in a $\mathrm{Y}$ site. To resolve the mixed occupancy of the $\mathrm{Y} 2 / \mathrm{Na} 1$ site, we first transformed the structure into a $\mathrm{P} 1$ basis using WebATOMS,${ }^{1}$ doubled the c-axis lattice parameter, and halved the $z$ position parameter of each atom, thus leaving the Cartesiancoordinate positions of all the atoms unaffected, but making an empty space in the doubled 
structure. We then filled that space with a copy of the existing atoms, shifted by $\vec{c} / 2$, resulting in a structure which was identical to the original but described using twice as many atoms in twice as big a cell. We then removed the Y2 site in the original half and the Na1 site in the copied half. The resulting crystal-structure description, with $\mathrm{Yb}$ in the $\mathrm{Y} 1$ site is as follows:

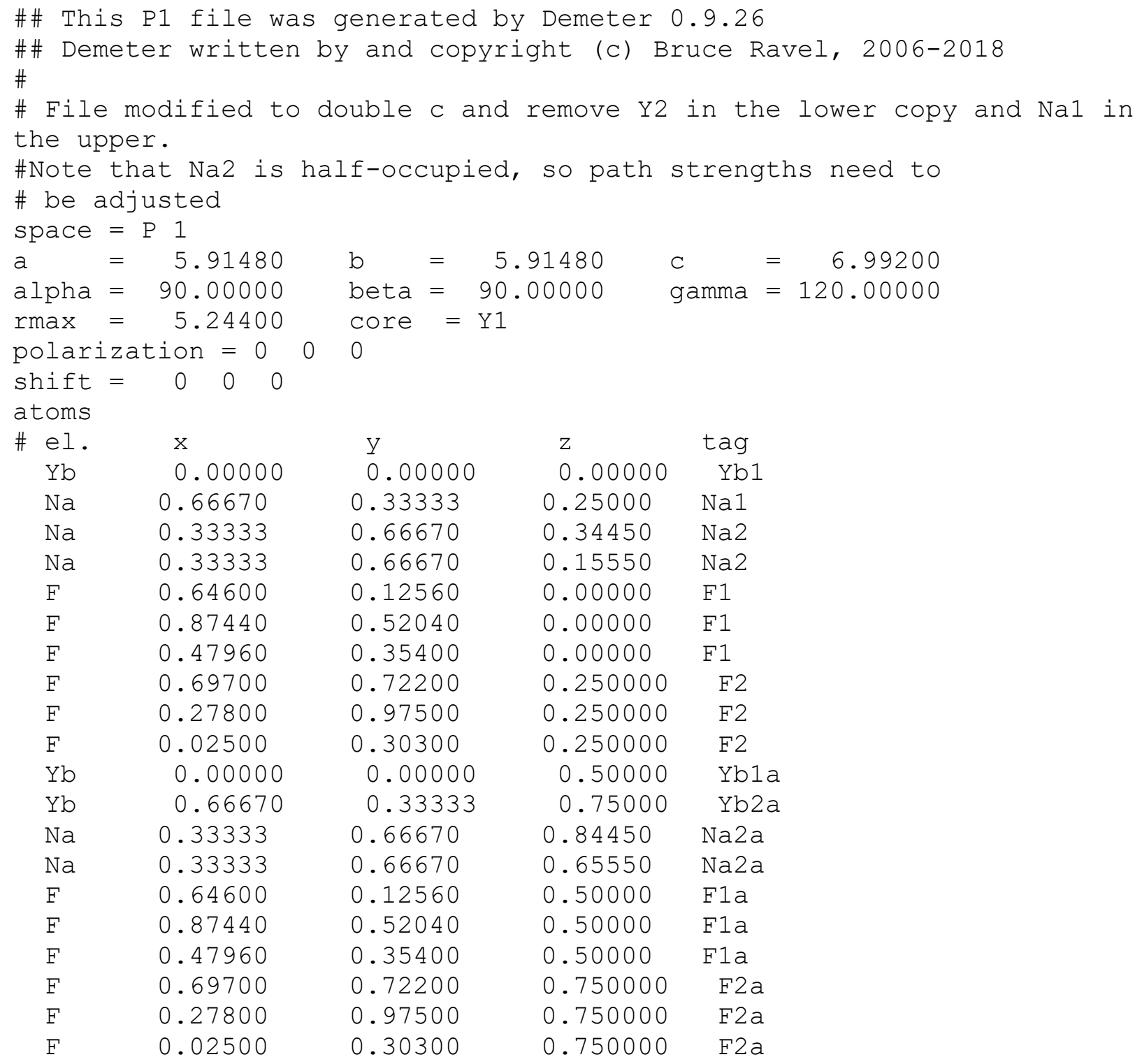

This table became the input for WebATOMS to make the feff.inp file. To address the vacancies on the $\mathrm{Na} 2$ sites, we manually halved all the path amplitudes involving this site.

\section{Fitting}

We tried fitting with $\mathrm{Yb}$ in $\mathrm{Y} 1$ and $\mathrm{Y} 2$ sites, but found that fitting $\mathrm{Yb}$ only in the $\mathrm{Y} 1$ position produced the lowest reduced chi-squared value. We ended up with 8 paths, ranging in distance from the nearest-neighbor fluorines at $2.45 \AA$ (undistorted structure) to a Na2 shell at $4.18 \AA$. 
The free parameters used were overall $\mathrm{E}_{0}$, overall $S_{0}^{2}$, distance shifts for the two $1 \mathrm{NN}$ fluorine shells, the $\Delta \sigma^{2}$ for these shells (equal for the two), the $\Delta \sigma^{2}$ for the higher shells, a distance shift relative to the reference structure for the $\mathrm{F}$ and $\mathrm{Y}$ atoms, and an independent one for the Na1 atom at $3.83 \AA$. We also tried freeing the occupancy for the Na2 sites, the distance of the Na2 shell at $3.58 \AA$ and the $\Delta \sigma^{2}$ for the Na2 site at $4.18 \AA$ but none of these helped the fit.

\section{Results}

We find that the EXAFS fits with our substituted $P \overline{6}$ model, but with most distances shorter than in the pure $\mathrm{Na}_{1.5} \mathrm{Y}_{1.5} \mathrm{~F}_{6}$ model, which makes sense as $\mathrm{Yb}^{3+}$ is a smaller ion than $\mathrm{Y}^{3+}$. The $\mathrm{Yb}$-F distances were 2.25,2.40 $\AA$ (error bar $0.02 \AA$, reference structure 2.454,2.458 $\AA$ ). The Na1 at a nominal distance of $3.84 \AA$ is instead at $3.78 \pm 0.03 \AA$, and the one at $4.12 \AA$ in the reference structure is found at $4.12 \pm 0.03 \AA$. The other distances are $0.05 \pm 0.02 \AA$ greater than in the reference structure.

\section{SI References}

(1) Ravel, B.; Newville, M. ATHENA, ARTEMIS, HEPHAESTUS: Data Analysis for X-Ray Absorption Spectroscopy Using IFEFFIT. J. Synchrotron Radiat. 2005, 12 (4), 537-541. https://doi.org/10.1107/S0909049505012719. 